\title{
Free Molecule Studies by Perturbed $\gamma-\gamma$ Angular Correlation: A New Path to Accurate Nuclear Quadrupole Moments
}

\author{
Heinz Haas $\odot,{ }^{1,2}$ Jens Röder, ${ }^{1,2}$ Joao G. Correia $\odot,{ }^{3,2}$ J. Schell@, ${ }^{4,2}$ Abel S. Fenta $\odot,{ }^{1}$ Reiner Vianden, ${ }^{5}$ \\ Emil M. H. Larsen $\odot,{ }^{6}$ Patrick A. Aggelund, ${ }^{6}$ Rasmus Fromsejer, ${ }^{6}$ Lars B. S. Hemmingsen $\odot,{ }^{6}$ \\ Stephan P. A. Sauer $\odot{ }^{6}$ Doru C. Lupascu, ${ }^{4}$ and Vitor S. Amaral ${ }^{1}$ \\ ${ }^{1}$ Department of Physics and CICECO, University of Aveiro, 3810-193 Aveiro, Portugal \\ ${ }^{2}$ EP Division CERN, 1211 Geneve-23, Switzerland \\ ${ }^{3} C 2 T N$, DECN, Instituto Superior Técnico, Universidade de Lisboa, Portugal \\ ${ }^{4}$ Institute for Materials Science and Center for Nanointegration, Duisburg-Essen (CENIDE), University of Duisburg-Essen, \\ 45141 Essen, Germany \\ ${ }^{5}$ Helmholtz-Institut für Strahlen- und Kernphysik, Universität Bonn, Nussallee 14-16, Bonn, Germany \\ ${ }^{6}$ Department of Chemistry, University of Copenhagen, 2100 Copenhagen, Denmark
}

(Received 12 December 2020; revised 22 January 2021; accepted 26 January 2021; published 11 March 2021)

\begin{abstract}
Accurate nuclear quadrupole moment values are essential as benchmarks for nuclear structure models and for the interpretation of experimentally determined nuclear quadrupole interactions in terms of electronic and molecular structure. Here, we present a novel route to such data by combining perturbed $\gamma-\gamma$ angular correlation measurements on free small linear molecules, realized for the first time within this work, with state-of-the-art ab initio electronic structure calculations of the electric field gradient at the probe site. This approach, also feasible for a series of other cases, is applied to $\mathrm{Hg}$ and Cd halides, resulting in $Q\left({ }^{199} \mathrm{Hg}, 5 / 2^{-}\right)=+0.674(17) b$ and $Q\left({ }^{111} \mathrm{Cd}, 5 / 2^{+}\right)=+0.664(7) b$.
\end{abstract}

DOI: 10.1103/PhysRevLett.126.103001

Introduction.-The knowledge of accurate nuclear moments is of importance both for our understanding of fundamental nuclear properties as well as for a quantitative interpretation of nuclear probe studies in matter. To determine these moments in hyperfine interaction experiments one measures the Larmor frequency $\nu_{L}=\mu B / I h$ or the quadrupole coupling constant $\nu_{Q}=e Q V_{z z} / h$. To obtain the magnetic moment $\mu$ one generally uses a known external field $B$. To obtain the nuclear quadrupole moment $Q$ from the observed $\nu_{Q}$ requires a theoretical calculation of the electric field gradient (EFG), $V_{x y}=\partial^{2} \Phi(\vec{r}) / \partial x \partial y$ of the electrostatic potential $\Phi(\vec{r})$ at the nuclear site. In recent years great progress has been made [1] to reliably determine $V_{z z}$, and therefore $Q$, for atomic and simple molecular systems, where measurements with stable (or very longlived) isotopes are available. These may then be used, after often difficult experimental nuclear quadrupole resonance spectroscopy studies [2], to normalize the abundant data for (generally short-lived) excited nuclear states, mostly obtained in metallic matrix systems [3]. For cases where the element of interest has no stable isotope with spin

Published by the American Physical Society under the terms of the Creative Commons Attribution 4.0 International license. Further distribution of this work must maintain attribution to the author(s) and the published article's title, journal citation, and DOI.
$I>1 / 2$, however, $(\mathrm{F}, \mathrm{Cd}, \mathrm{Sn}, \mathrm{Pb}$, e.g. $)$ this procedure fails. With this work, we have devised a route to accurate determination of $Q$ for short-lived excited states by measuring $\nu_{Q}$ in free molecules using the nuclear spectroscopy technique of perturbed $\gamma-\gamma$ angular correlation (PAC) [4].

The PAC method has over the last 50 years become an established tool in materials physics for investigating magnetic fields and EFGs in condensed matter, widely used in solid-state- [5], semiconductor- [6], surface- [7], and bio-physics [8] research. It has, however, never before been applied to free molecules in the gas phase.

During a PAC experiment, shown schematically in Fig. 1, a periodic modulation of the coincidence count rate is measured as a function of delay time between $\gamma_{1}$ and $\gamma_{2}$ in a form depending on the interdetector angle. Analyzing such data, one forms the (properly normalized) ratio of count rates $W$ at $180^{\circ}$ and $90^{\circ}$ interdetector angles

$$
R(t)=2 \frac{W(180, t)-W(90, t)}{W(180, t)+2 W(90, t)}=A_{22}^{\exp } G_{22}(t)
$$

to eliminate the time effects of the exponential decay. After correction of the nuclear angular correlation coefficient $A_{22}$ for finite detector and sample size, typically reduced by $5 \%$ to $15 \%$, this function yields the perturbation function $G_{22}(t)$ that contains all the information about the environmental effects. 


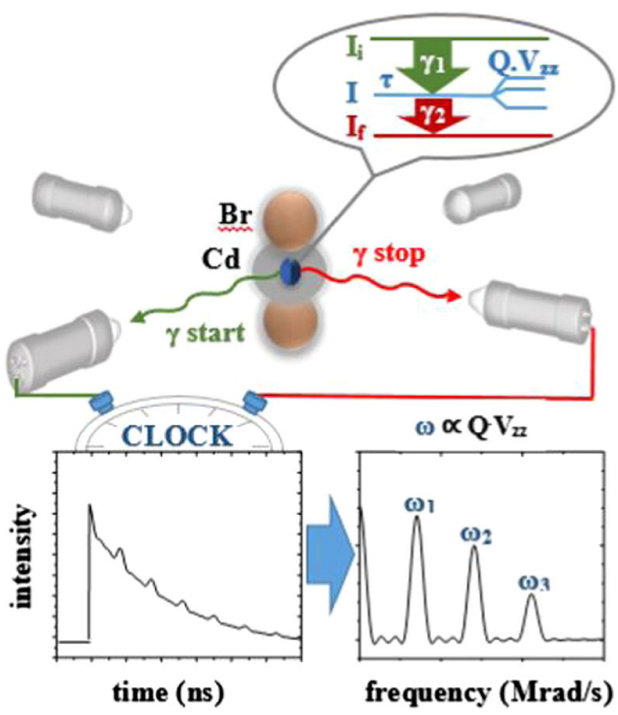

FIG. 1. Schematic drawing of the PAC experiment on a free molecule. The $\mathrm{Cd}$ nucleus with quadrupole moment $Q$ (dark blue) interacts with the EFG $\left(V_{z z}\right)$ created by the Cd electron distribution (gray) modified by bonding to the bromine atoms (brown). This is observed by the modulation of the time distribution of the two $\gamma$ rays emitted in decay of ${ }^{111 \mathrm{~m}} \mathrm{Cd}$. The energy splitting of the nuclear intermediate state is directly seen as transition frequencies $(\omega)$ in the Fourier transform.

Results in solids have recently been routinely augmented by theoretical calculations using density functional techniques [9] to obtain $V_{z z}$. It has become apparent, however, that very precise values of the EFG, needed for a reliable determination of $Q$, require high level ab initio quantum chemistry calculations, only possible for rather small molecules. Moreover, calculation of the EFG in the condensed phase requires that the surroundings of the probe atom in the crystal is well known, in addition compromising accurate calculation of the EFG. The PAC data recorded for gas phase molecules may therefore be applied in combination with state-of-the-art first principle electronic structure calculations to derive accurate values for the relevant nuclear quadrupole moments.

Though the application of the PAC method to gases has been discussed even in the earliest reports, essentially no experiments with clear results have been performed as yet. No measurements of free molecules have been successful to date, early attempts in Berkeley [5,10] and Bonn [11] having failed due to experimental difficulties. We have now overcome these technical problems and studied the nuclear quadrupole interaction at ${ }^{199} \mathrm{Hg}$ and ${ }^{111} \mathrm{Cd}$ in a few simple linear halide molecules. The new experimental data, combined with state-of-the-art quantum chemical calculations, have resulted in an independent and accurate determination of the nuclear quadrupole moments $Q$ involved. Strictly speaking our results are only for the absolute values of $Q$, the sign being known. Moreover, in comparison with earlier data of the EFG at ${ }^{199} \mathrm{Hg}$ in the corresponding molecular solids [12], our measurements form a unique test case for intermolecular interaction effects.

The basic idea of our approach originates from the fact that in a linear molecule the principal axis of the EFG is by symmetry along the molecular axis and the rotational angular momentum $J$ is perpendicular to this for all states. The component of the EFG tensor along $J$ is thus equal to $-1 / 2$ times that along the molecular axis. The nuclear spin I being much smaller than $J$ for practically all thermally populated rotational states leads to a total $\boldsymbol{F}=\boldsymbol{J}+\boldsymbol{I}$ essentially aligned with $J$. The resulting perturbation function is then expected to be very similar for all $J$ states, highly resembling the well-known one for a randomly oriented axially symmetric EFG. The dominating rotational angular momenta as calculated using the known molecular geometry [13] (see Table SM2) are in the range of $20<$ $J<200$ for the cases considered here.

The technical challenge to be overcome is the production of a highly diluted gas sample of the radioactive molecules such that no intermolecular collisions occur during the measurement time. This is typically a few half-lives of the intermediate nuclear state, $2.47 \mathrm{~ns}$ in the case of ${ }^{199} \mathrm{Hg}$ $(I=5 / 2)$ and $84.5 \mathrm{~ns}$ in the case of ${ }^{111} \mathrm{Cd}(I=5 / 2)$. Decay details are shown in Fig. SM1 of Supplemental Material (SM) [14]. The pure beams of the ${ }^{199} \mathrm{~m} H g$ (half-life $42.7 \mathrm{~min}$ ) and ${ }^{111 \mathrm{~m}} \mathrm{Cd}$ (half-life $48.5 \mathrm{~min}$ ) parent isotopes produced at the ISOLDE facility at CERN [15] are ideally suited for such an experiment. Samples of the ${ }^{199 \mathrm{~m}} \mathrm{Hg}$ and ${ }^{111 \mathrm{~m}} \mathrm{Cd}$ halides with a small amount of stable carrier were produced by a chemical procedure following isotope implantation into frozen water (see SM [14]) and sealed in glass ampoules. These were then measured at elevated temperatures (and at room temperature for comparison) in a standard 4-detector setup of $\mathrm{LaBr}_{3}$ detectors in cross geometry with digital electronics [16].

The analysis of the present experimental results was augmented by state-of-the-art quantum chemistry calculations for the free molecules using the DIRAC15 code [17] and density functional calculations using WIEN2k [18] for solids as described below.

Proof of principle: $\mathrm{Hg}$ halides.-Optimal conditions for a PAC measurement are presented by a short intermediate state half-life and heavy molecules as realized for the $\mathrm{Hg}$ halides. Figure 2 shows the resulting normalized perturbation functions for the molecular solids, the isolated gas molecules, and the concentrated gas. For the solid samples they show the well-known characteristic periodic pattern for $I=5 / 2$ with frequency contributions as $3 \nu_{0}, 2 \nu_{0}, 1 \nu_{0}$, and $0 \nu_{0}\left(\nu_{0}=3 \nu_{Q} / 20\right)$. For the dilute gases a similar pattern at about double the period is observed, related to the EFG component perpendicular to the molecular axis. Within the experimental accuracy the data may be represented with the simple function including an exponential damping term. This, however, would lead to a frequency error in the $1 \%$ range as discussed below. As a further 


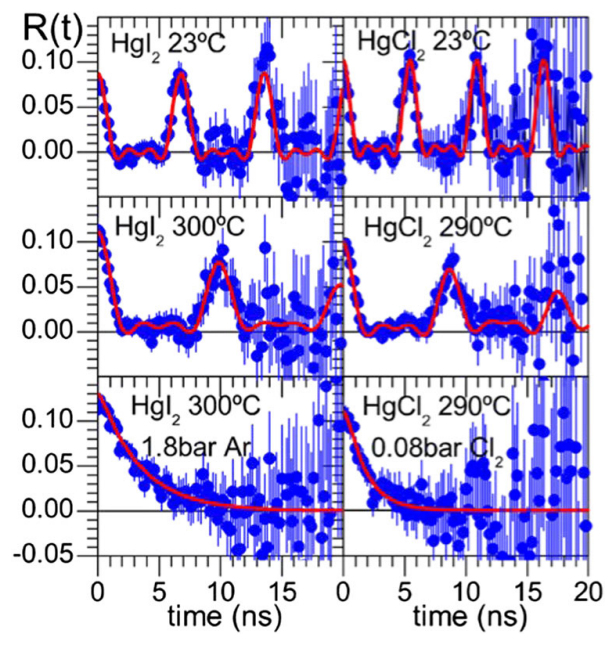

FIG. 2. Observed PAC patterns for $\mathrm{Hg}$ halides at room temperature (top), in the gas phase (middle), and with additional other gas (bottom) (blue: data; red: theory).

check, samples with additional buffer gas added were produced that show the expected quasiexponential anisotropy decay [19] for molecules undergoing several reorientation collisions during the time of measurement. A more systematic study of this effect using all noble gases will be described separately.

Using earlier detailed calculations of the EFG for the $\mathrm{Hg}$ halides [20,21] our experimental results now allow an independent determination of the nuclear quadrupole moment for the ${ }^{199} \mathrm{Hg} 5 / 2^{-}$state as $Q=0.674(17) b$, see Table I. The number has to be compared with the value of $Q=0.677(13) b$ as obtained from recent theoretical atomic physics calculations [22] for ${ }^{201} \mathrm{Hg}$ combined with the experimental ratios from earlier solid-state studies $[12,23]$. The complete agreement is a decisive confirmation of our approach. Earlier data for $Q$ of excited states in some other Hg isotopes [24] have been reanalyzed with this result in Table SM4 [14]. It should be noted that this renormalization could be made even more precise with the availability of a better value for $\nu_{Q}$ in solid $\mathrm{Hg}$, either from PAC using ${ }^{199} \mathrm{Hg}$ or from nuclear quadrupole resonance spectroscopy using ${ }^{201} \mathrm{Hg}$.

We further point out that the quadrupole interaction frequencies obtained here for the solid samples are in full accord with the earlier data [12]. The present slightly higher value for $\mathrm{HgI}_{2}$, measured at room temperature in a metastable state of the solid, when compared to the literature value at $407 \mathrm{~K}$, is to be expected as a consequence of the typical EFG temperature dependence in solids.

Nuclear quadrupole moment: Cd halides.-The original motivation to initiate the present experimental program was to obtain a precise number for the nuclear quadrupole moment of the ${ }^{111} \mathrm{Cd} 5 / 2^{+}$state. This value is essential to extract the correct result of $Q$ for a number of nuclear excited states in the $\mathrm{Cd}$ chain from the measured interaction frequencies and future planned experiments. Since this state is also used in most PAC experiments, it is obvious that a correct $Q$ is as well essential to get the EFG values from the data. It had become evident over the last years, however, that the value obtained by standard density functional methods [25,26] applied to solid-state data suffers from systematic errors. The use of hybrid density functional techniques has changed this value considerably, as was the case for some other nuclei [27]. In a recent work precision state-of-the-art quantum chemistry calculations of the EFG have been applied to the Cd-dimethyl molecule [28]. Unfortunately, the molecular $\nu_{Q}$ in this case had to be obtained from the value measured in the solid, introducing an additional uncertainty. For an accurate result it thus became evident that reliable $\nu_{Q}$ values for some simple free $\mathrm{Cd}$ molecules had to be obtained and combined with precision EFG calculations.

Because of the longer lifetime of the intermediate PAC state of ${ }^{111} \mathrm{Cd}$ and the higher boiling points of the $\mathrm{Cd}$ halides the corresponding experiments were much more challenging than for ${ }^{199} \mathrm{Hg}$. In particular, for $\mathrm{CdCl}_{2}$ only a small temperature interval turned out to be available. Figure 3 shows the

TABLE I Summary of experimental data and analysis results. Row 1: directly observed interaction frequencies (statistical errors). Row 2: theoretical corrections due to $\mathbf{J}+\mathbf{I}$ coupling, centrifugal stretching and vibration (see SM [14]). Row 3: free molecule interaction frequencies obtained as $2 \nu_{\mathrm{obs}}\left(1-\mathrm{corr}_{\mathrm{th}}\right)$. Row 4: theoretical EFG at CCSD-T level (typical error $1 \%$ assumed). Row 5: calculated nuclear quadrupole moments for ${ }^{199} \mathrm{Hg}$ and ${ }^{111} \mathrm{Cd} \mathrm{PAC}$ states.

\begin{tabular}{lccccc}
\hline \hline & $\mathrm{HgCl}_{2}$ & $\mathrm{HgI}_{2}$ & $\mathrm{CdCl}_{2}$ & $\mathrm{CdBr}_{2}$ & $\mathrm{CdI}_{2}$ \\
\hline$\nu_{\text {obs }}[\mathrm{MHz}]^{\mathrm{a}}$ & $757(16)$ & $673(13)$ & $345.7(40)$ & $321.0(15)$ & $298.8(8)$ \\
corr $_{\text {th }}[\%]^{\mathrm{a}}$ & $+1.15(20)$ & $-0.14(20)$ & $-1.58(40)$ & $-1.74(40)$ & $-2.48(40)$ \\
$\nu_{Q}^{\text {mol }}[\mathrm{MHz}]^{\mathrm{a}}$ & $1497(34)$ & $1348(29)$ & $702(10)$ & $653(5)$ & $612(3)$ \\
$V_{z z, \text { th }}[\mathrm{au}]$ & $9.51(10)^{\mathrm{b}}$ & $8.46(9)^{\mathrm{b}, \mathrm{d}}$ & $4.51(5)^{\mathrm{a}}$ & $4.22(4)^{\mathrm{a}}$ & $3.90(4)^{\mathrm{a}}$ \\
\hline$Q_{\text {cal }}[b]^{\mathrm{a}}$ & $0.670(17)$ & $0.678(15)$ & $0.663(10)$ & $0.659(8)$ & $0.668(7)$ \\
$\nu_{Q}^{\text {sol }}[\mathrm{MHz}]$ & $1235(12)^{\mathrm{a}}$ & $980(10)^{\mathrm{a}}$ & $48.7(10)^{\mathrm{c}}$ & $24.6(5)^{\mathrm{c}}$ & $9.7(8)^{\mathrm{c}}$ \\
\hline \hline
\end{tabular}

${ }^{\mathrm{a}}$ Present work.

${ }^{b}$ Ref. [20].

${ }^{\mathrm{c}}$ Ref. [5].

${ }^{\mathrm{d}}$ Corrected for $R_{\text {exp }}$ discrepancy. 


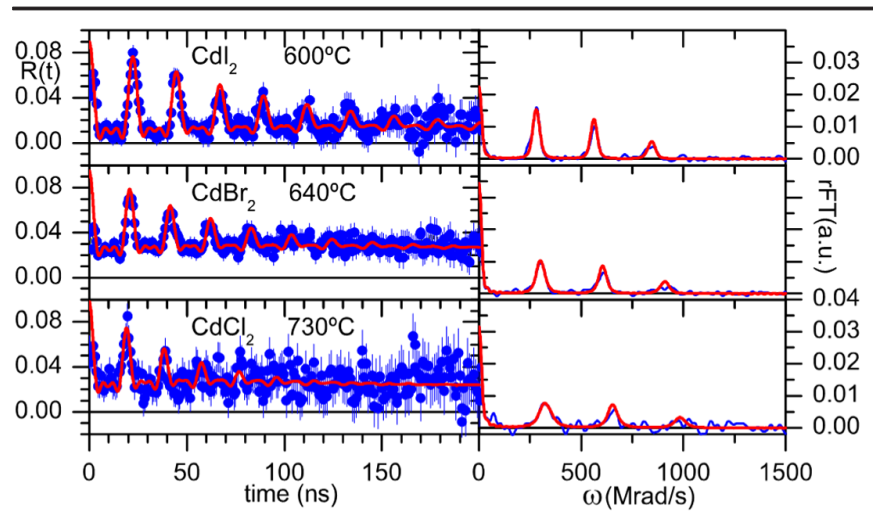

FIG. 3. Observed PAC patterns and Fourier transforms for $\mathrm{Cd}$ halides in the gas phase (blue: data; red: theory). The Fourier transformations demonstrate that just taking three frequency components with ratio $1: 2: 3$ is a good approximation to the complex perturbation function shown.

spectra obtained, allowing a precise determination of the interaction frequencies. The included Fourier transforms of the data and the theory function clearly show the contributions of essentially three harmonics of the interaction frequency as expected from our basic concept. In Table I the results, including the small corrections outlined below, are combined with the theoretical EFG values obtained in quantum chemistry calculations at the full four-component relativistic level using the coupled cluster single-doubletriple (CCSD-T) method. To obtain the necessary theoretical data for the vibrational corrections, density functional calculations with the Perdew-Burke-Ernzerhof hybrid (PBE0) functional [29] were performed for molecules deformed by symmetric stretching and bending. Dyall's cv3z basis set [30] was used in all calculations.

The three independent determinations of $Q$ agree with each other within the error bars. This now sets the value of the nuclear quadrupole moment of the ${ }^{111} \mathrm{Cd}$ PAC state on a solid footing, and, along with it, improves the accuracy of $Q$ for the isomeric states of other Cd isotopes [24], related to it in most cases by rather precise experimental ratios, corrected with the known temperature dependence of the EFG [31] (see Table SM4 [14]). In particular, the theoretically predicted saturation of $Q$ for $11 / 2^{-}$states in the most neutron deficient Cd isotopes [28] is confirmed.

Analysis and results.-For an exact treatment of the perturbation function a full quantum-mechanical description of the coupling between the nuclear spin I and the rotational angular momentum $J$ is needed. The basic relationships have been worked out a long time ago $[4,32]$, but have never been applied to a similar situation. The energy levels for $\mathbf{J}+\mathbf{I}$ coupling are [32]

$$
\begin{gathered}
E_{F}=\nu_{Q} \frac{6[K(K+1)-(4 / 3) I(I+1) J(J+1)]}{(I)(2 I-1)(J)(2 J-1)}, \\
K=F(F+1)-I(I+1)-J(J+1) .
\end{gathered}
$$

The perturbation function is given by [4]

$$
\begin{aligned}
G_{22}(t)= & \sum_{F, F^{\prime}}(2 F+1)\left(2 F^{\prime}+1\right)\left\{\begin{array}{l}
F F^{\prime} 2 \\
I I J
\end{array}\right\}^{2} \\
& \times \exp \left[-(i / \hbar)\left(E_{F-} E_{F^{\prime}}\right) t\right] \\
= & \sum_{F, F^{\prime}} a_{F F^{\prime}} \cos \left[(1 / \hbar)\left(E_{F-} E_{F^{\prime}}\right) t\right] .
\end{aligned}
$$

As demonstrated in detail in Figs. SM3 and SM4 [14], this leads for $J>10$ only to a slight splitting and shift of the four contributing perturbation frequencies $0 \nu_{0}, 1 \nu_{0}, 2 \nu_{0}$, and $3 \nu_{0}\left(\nu_{0}=3 \nu_{Q} / 20\right)$ at $J$ infinite as well as a minor change of their relative amplitude contributions, marginally different for every $J$.

In addition, changes of the EFG due to the centrifugal stretching of the molecules and excitation of vibrational states at the measuring temperature must be incorporated. As also illustrated in detail in SM [14], these small contributions have been determined in a straightforward but rather complex way applying the standard molecular physics approach $[33,34]$, making use of the radius and angular dependence of the EFG and total energy as calculated with the quantum chemical code. In addition, the effects of rest-gas collisions lead to a further damping that can be treated within a stochastic model [35-37]. The final fit results are presented in Table I.

Gas-solid EFG shift.-Another interesting outcome of the present experiment is the change of the EFG in going from the isolated molecule to the condensed molecular solid. This is a direct consequence of the wave function change by the intermolecular interaction. Only for the simple dihalogen molecules are there equivalent data already available for checking solid-state calculations [38] of the effects of the intermolecular bonding on the electronic charge distribution mirrored by the EFG. A preliminary theoretical analysis of the measured change in $V_{z z}$ by $-17.5 \%$ for $\mathrm{HgCl}_{2}$ (see Table I) gives (a) temperature effect in the solid (experimental [23]): $-2.5 \%$; (b) different $\mathrm{Hg}-\mathrm{Cl}$ distance in solid [39] and molecule [13,40] (CCSD-T): $-6.3 \%$; (c) intermolecular interaction (DFT LDA): $-7.4 \%$.

Conclusions. - In summary, we have demonstrated that the nuclear technique of perturbed $\gamma-\gamma$ angular correlation is well suited to study the quadrupole hyperfine interaction in free molecules in the gas phase, allowing the determination of precise nuclear quadrupole moments (see Fig. 4). Such results also offer a unique benchmark for quantum chemical calculations. Further on, when compared to measurements of the molecules in the solid state, they permit to experimentally investigate the direct effect of intermolecular interactions on the local electron distribution in condensed matter.

Outlook.-Similar experiments to the present ones are envisioned using ${ }^{80 \mathrm{~m}} \mathrm{Br},{ }^{204 \mathrm{~m}} \mathrm{~Pb}$, and possibly even ${ }^{119 \mathrm{~m}} \mathrm{Sn}$, 


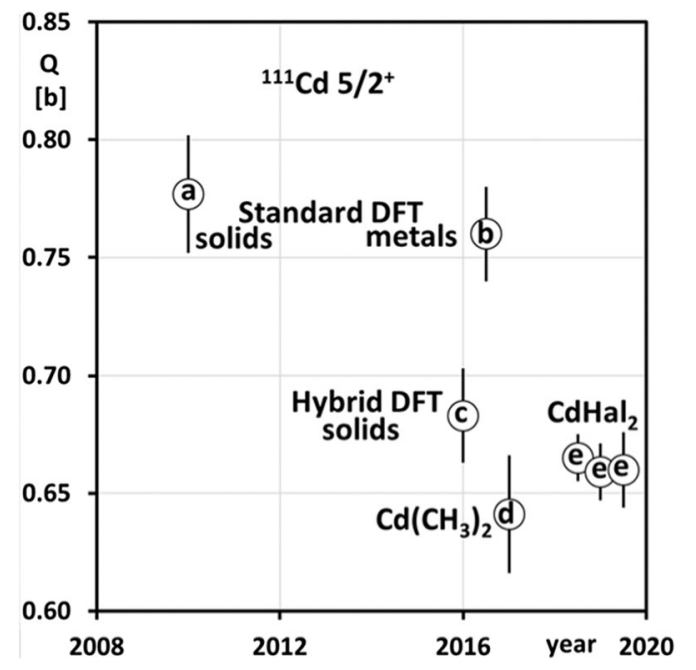

FIG. 4. Resulting $Q$ for ${ }^{111} \mathrm{Cd}\left(5 / 2^{+}\right)$from recent $\mathrm{EFG}$ calculations using various methods (a) Ref. [25], (b) Ref. [26], (c) Ref. [27], (d) Ref. [28], (e) present work.

where the primary goal would first be to obtain more reliable nuclear quadrupole moments than hitherto available.

It would also be possible to investigate molecules with more complex geometry using the new technique, though the required calculation for the proper perturbation function will be more complicated and generally not result in a quasiperiodic structure.

The present pilot experiments also open up the possibility to study charged molecular states using $\beta^{-}$emitting sources, where the production of a single charge state is to be expected upon the radioactive element transmutation, PAC becoming a unique method to perform spectroscopic measurements on isolated charged molecules.

The assistance of the CERN technical staff, Bernard Crepieux and Miranda von Stenis in particular, was essential for the success of our experiments. H. H. would like to thank S. Kranawetter and family for their hospitality during his work at CERN. Correspondence with M. Hargittai (Budapest) was helpful in choosing the appropriate molecular geometries. This work was funded by German Federal Ministry of Education and Research (BMBF) through Contract No. 05K16PGA, the Belgian KU Leuven contract (SF/18/008), the Portuguese Foundation for Science and Technology (Project No. CERN/FIS-PAR/0005/2017, and CICECO-Aveiro Institute of Materials, UIDB/50011/2020 \& UIDP/50011/2020). The EU Horizon 2020 Framework supported ISOLDE beam times through Grant No. 654002 (ENSAR2).

[1] P. Pyykkö, Year-217 nuclear quadrupole moments, Mol. Phys. 116, 1328 (2018).

[2] E. A. C. Lucken, Nuclear Quadrupole Coupling Constants (Academic Press, London, 1969).
[3] R. Vianden, Electric field gradients in metals, Hyperfine Interact. 35, 1077 (1987).

[4] H. Frauenfelder and R. M. Steffen, Angular correlations, in, Alpha-, Beta- and Gamma-Ray Spectroscopy, edited by K. Siegbahn (NORTH HOLLAND, Amsterdam 1965), pp. 997-1198.

[5] H. Haas and D. A. Shirley, Nuclear quadrupole interaction studies by perturbed angular correlations, J. Chem. Phys. 58, 3339 (1973).

[6] D. Forkel-Wirth, Radioactive ion beams in solid state physics, Phil. Trans. R. Soc. A 356, 2137 (1998).

[7] H. Haas, Surface physics with radioactive ion beams, Hyperfine Interact. 84, 69 (1994).

[8] L. Hemmingsen, K. N. Sas, and E. Danielsen, Biological applications of perturbed angular correlation of $\gamma$-ray spectroscopy, Chem. Rev. 104, 4027 (2004).

[9] P. Blaha, P. Dufek, K. Schwarz, and H. Haas, Calculation of electric hyperfine interaction parameters in solids, Hyperfine Interact. 97/98, 3 (1996).

[10] H. H. Rinneberg and D. A. Shirley (private communication).

[11] F. Reuschenbach, Zeitlich differentielle gestörte $\gamma$ - $\gamma$ Winkelkorrelationsuntersuchungen am freien $\mathrm{CdCl}_{2}$. Diplomarbeit, Universität Bonn, 1977.

[12] W. Tröger, T. Butz, P. Blaha, and K. Schwarz, Nuclear quadrupole interaction of ${ }^{199 \mathrm{~m}} \mathrm{Hg}$ in mercury(I) and mercury (II) halides, Hyperfine Interact. 80, 1109 (1993).

[13] M. Hargittai, Molecular structure of metal halides, Chem. Rev. 100, 2233 (2000).

[14] See Supplemental Material at http://link.aps.org/ supplemental/10.1103/PhysRevLett.126.103001 for details of sample preparation and data analysis method.

[15] R. Catherall et al., The ISOLDE facility, J. Phys. G 44, 094002 (2017).

[16] C. Herden, J. Röder, J. A. Gardner, and K. D. Becker, Fully digital time differential perturbed angular correlation (TDPAC) spectrometer, Nucl. Instrum. Methods Phys. Res., Sect. A 594, 155 (2008).

[17] H. J. A. Jensen et al., DIRAC, a relativistic $a b$ initio electronic structure program (Release DIRAC15) (2015), see http://diracprogram.org.

[18] P. Blaha, K. Schwarz, G. K. H. Madsen, D. Kwasnicka, and J. Luitz, WIEN2k, An Augmented Plane Wave +Local Orbitals Program for Calculating Crystal Properties (Karlheinz Schwarz, Techn. Universität Wien, Austria, 2001).

[19] A. Abragam and R. V. Pound, Influence of electric and magnetic fields on angular correlations, Phys. Rev. 92, 943 (1953).

[20] V. Arcisauskaite, S. Knecht, S. P. A. Sauer, and L. Hemmingsen, Electric field gradients in $\mathrm{Hg}$ compounds: Molecular orbital (MO) analysis and comparison of 4-component and 2-component (ZORA) methods, Phys. Chem. Chem. Phys. 14, 16070 (2012).

[21] V. Arcisauskaite, S. Knecht, S. P. A. Sauer, and L. Hemmingsen, Fully relativistic coupled cluster and DFT study of electric field gradients at $\mathrm{Hg}$ in ${ }^{199} \mathrm{Hg}$ compounds, Phys. Chem. Chem. Phys. 14, 2651 (2012).

[22] J. Bieron, P. Pyykkö, and P. Joensson, Nuclear quadrupole moment of ${ }^{201} \mathrm{Hg}$, Phys. Rev. A 71, 012502 (2005).

[23] H. G. Dehmelt, H. G. Robinson, and W. Gordy, Nuclear quadrupole resonance of $\mathrm{Hg}^{201}$, Phys. Rev. 93, 480 (1954). 
[24] N. J. Stone, Table of nuclear electric quadrupole moments, At. Data Nucl. Data Tables 111, 1 (2016) and references therein.

[25] H. Haas and J. G. Correia, The quadrupole moments of Cd and $\mathrm{Zn}$ isotopes-An update, Hyperfine Interact. 198, 133 (2010).

[26] L. Errico, K. Lejaeghere, J. Runco, S. N. Mishra, M. Rentería, and S. Cottenier, Precision of electric-field gradient predictions by density functional theory and implications for the nuclear quadrupole moment and its error bar of the ${ }^{111} \mathrm{Cd}$ $245 \mathrm{keV} \mathrm{5/2^{+ }}$ level, J. Phys. Chem. C 120, 23111 (2016).

[27] H. Haas, M. B. Barbosa, and J. G. Correia, The quadrupole moments of $\mathrm{Cd}$ and $\mathrm{Zn}$ isotopes-An apology, Hyperfine Interact. 237, 115 (2016).

[28] H. Haas et al., Quadrupole moments of $\mathrm{Cd}$ and $\mathrm{Zn}$ nuclei: When solid-state, molecular, atomic, and nuclear theory meet, Euro. Phys. Lett. 117, 62001 (2017).

[29] J. P. Perdew, M. Ernzerhof, and K. Burke, Rationale for mixing exact exchange with density functional approximations, J. Chem. Phys. 105, 9982 (1996).

[30] K. G. Dyall, Relativistic double-zeta, triple-zeta, and quadruple-zeta basis sets for the $4 d$ elements Y-Cd, Theor. Chem. Acc. 117, 483 (2007).

[31] J. Bleck, R. Butt, H. Haas, W. Ribbe, and W. Zeitz, In-Beam Measurements of the Nuclear Quadrupole Interaction in Cadmium Metal, Phys. Rev. Lett. 29, 1371 (1972).
[32] C. Schwarz, Theory of hyperfine structure, Phys. Rev. 97, 380 (1955).

[33] G. Herzberg, Molecular Spectra and Molecular Structure (Van Norstrand Reinhold Company, New York, 1945, 1950), Vols. I and II.

[34] A. D. Buckingham, Temperature-dependent chemical shifts in the NMR spectra of gases, J. Chem. Phys. 36, 3096 (1962).

[35] S. Dattagupta, Study of time-dependent hyperfine interactions by PAC, Mössbauer effect, $\mu \mathrm{SR}$ and NMR: A review of stochastic models, Hyperfine Interact. 11, 77 (1981).

[36] E. Danielsen and R. Bauer, Analysis of perturbed angular correlation spectra of metal ions bound to proteins with rotational correlation times in the intermediate region, Hyperfine Interact. 62, 311 (1991).

[37] R. M. Lynden-Bell, Perturbation of the angular correlation of $\gamma$-rays by molecular motion, Mol. Phys. 21, 891 (1971).

[38] H. Haas, Quadrupole interaction in the solid halogensA new (not final) look, Hyperfine Interact. 197, 17 (2010).

[39] V. Subramanian and K. Seff, Mercuric chloride, a redetermination, Acta Crystallogr. Sect. B 36, 2132 (1980).

[40] K. Kashiwabara, S. Konaka, and M. Kimura, Electron diffraction investigation of gaseous mercury(II) chloride, Bull. Chem. Soc. Jpn. 46, 410 (1973). 